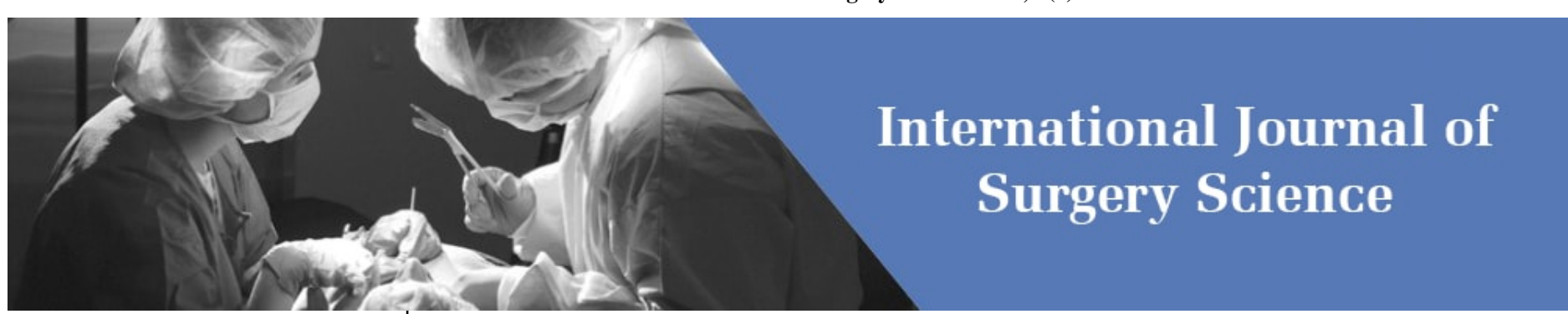

E-ISSN: 2616-3470

P-ISSN: 2616-3462

(C) Surgery Science

www.surgeryscience.com

2021; 5(4): 100-102

Received: 09-07-2021

Accepted: 16-09-2021

Mukul Sharma

Senior Resident, Department of General Surgery, Indira Gandhi

Medical College, Shimla, Himacha

Pradesh, India

\section{Yashika Sharma}

Dr. Rajendra Prasad Government

Medical College, Tanda, Kangra,

Himachal Pradesh. India

\section{Dhruv Sharma}

Department of General Surgery, Indira Gandhi Medical College,

Shimla, Himachal Pradesh, India

Arun Chauhan

Department of General Surgery,

Indira Gandhi Medical College,

Shimla, Himachal Pradesh, India

Arun Kumar Gupta

Department of General Surgery,

Indira Gandhi Medical College,

Shimla, Himachal Pradesh, India

\section{Jagdish Gupta}

Department of General Surgery, Indira Gandhi Medical College,

Shimla, Himachal Pradesh, India

\section{Blood sugar level as an indicator of necrosis in patients of gallstone induced acute pancreatitis}

\author{
Mukul Sharma, Yashika Sharma, Dhruv Sharma, Arun Chauhan, Arun \\ Kumar Gupta and Jagdish Gupta
}

DOI: https://doi.org/10.33545/surgery.2021.v5.i4b.769

Abstract

Acute necrotizing pancreatitis is a severe disease with more mortality as compared to interstitial pancreatitis. A screening test which is readily available for the detection of pancreatic necrosis would aid a lot in the early management of gallstone induced acute necrotizing pancreatitis. This study was conducted in patients of gallstone induced acute pancreatitis presenting to the emergency department of Indira Gandhi medical college, Shimla, Himachal Pradesh. 61 patients were included in this study. Initial blood sugar levels were compared to the occurrence of pancreatic necrosis. We concluded that normal or low blood sugar level reflects absence of necrosis in acute pancreatitis. Therefore, it may be used to rule out the possibility of acute necrotizing pancreatitis. This will help a lot in management of such patients in areas where resources are limited and scarce.

Keywords: Necrotizing pancreatitis, gallstone induced pancreatitis, blood sugar level and pancreatic necrosis

\section{Introduction}

Acute necrotizing pancreatitis is a severe disease with more mortality as compared to interstitial pancreatitis ${ }^{[1]}$. It is defined as existence of tissue necrosis in the pancreatic parenchyma or the peripancreatic tissues. Necrotizing pancreatitis can get infected and further lead to increased morbidity and mortality ${ }^{[2]}$. Contrast enhanced computed tomography (CECT) is gold standard for detection of pancreatic or peripancreatic necrosis. However, this investigation is not available everywhere and is not recommended in the first 72 hours as it may underestimate the extent of necrosis. Therefore, a screening test which is readily available for the detection of pancreatic necrosis would aid a lot in the early management of such patients. Gallstones are the most common cause of acute pancreatitis ${ }^{[3]}$ and we routinely manage cases of acute gallstone induced pancreatitis in our institute. So, our study evaluates the use of blood sugar levels on arrival of the patient to the hospital for early detection of necrosis in the acute gallstone induced pancreatitis.

\section{Materials and Methods}

This study was conducted in the department of general surgery at Indira Gandhi Medical College, Shimla, Himachal Pradesh. Patients of acute pancreatitis presenting to the emergency department, with presence of gallstones on ultrasound abdomen were included in the study. This study was carried out between June 2016 to June 2017. Diabetic patients, pregnant females and patients below 18 years of age were excluded from the study. A written informed consent was taken from all patients before inclusion. A total of 61 patients were encompassed in this study.

\subsection{Data collection}

Blood samples were sent to the hospital laboratory within 1 hour of arrival of the patients with acute gallstone induced pancreatitis to the emergency department. Blood sugar was recorded for each individual. A CECT abdomen was performed after 72 hours of onset of pain for all these patients to look for evidence of necrosis in the pancreas.

\subsection{Outcome of interest}

Primary outcome was presence of necrosis either in the pancreatic or peripancreatic tissues detected on CECT abdomen. 
The recorded initial blood sugar levels were then compared to the occurrence of pancreatic necrosis. This would give us an insight into the value of blood sugar levels as a marker and screening investigation for the detection of necrotizing pancreatitis.

\subsection{Statistical analysis}

Blood sugar level was considered as a continuous variable. All patients were divided into two groups based on presence or absence of necrosis (necrosis and no necrosis group). Receiver operator characteristic (ROC) curve for blood sugar level was plotted and area under the curve (AUC) was calculated for detection of pancreatic necrosis. Cut-off for blood sugar level was defined based on the ROC curve. 2x2 contingency table was made based on this cut off value. Sensitivity, specificity, negative predictive value (NPV) and positive predictive value (PPV) was calculated for the same. Chi-square test was applied and p-value was computed. A p-value of $<0.05$ was considered statistically significant. All data was analysed using Epi info version 7.2.3.1 software.

\section{Results}

Total 61 patients of gallstone induced acute pancreatitis were included in this study. All these patients were not a known case of diabetes mellitus. These consisted of 46 females and 15 males, making male to female ratio of around 3:1. On performing CECT abdomen in all patients after 72 hours, 14 were found to have pancreatic necrosis. The mean blood sugar in patients with necrosis was $163 \pm 73.4 \mathrm{mg} / \mathrm{dL}$ and in without necrosis was $127 \pm 56.4 \mathrm{mg} / \mathrm{dL}$. This difference was not significant (p-value 0.053). (Table 1)

ROC curve was plotted for blood sugar level detecting necrotizing pancreatitis. Area under the curve was 0.689 (Fig. 1). Based on the curve cut-off was defined as $140 \mathrm{mg} / \mathrm{dL} .2 \times 2$ contingency table was drawn. Based on this sensitivity, specificity, PPV and NPV were 57.14\%, 78.72\%, 44.44\% and 86.05\%. Chi-square test was applied and p-value was 0.010 which was significant.

Table 1: Comparison of demographics and blood sugar level in patients of necrotizing and interstitial gallstone induced acute pancreatitis

\begin{tabular}{|c|c|c|c|c|}
\hline & Overall & Necrotizing Pancreatitis & Interstitial Pancreatitis & p-value \\
\hline Age (Years) & $51.9 \pm 16.1$ & $54.5 \pm 16.0$ & $51.1 \pm 16.2$ & $0.493^{*}$ \\
\hline Sex (Female) & $46(75.4 \%)$ & $10(71.4 \%)$ & $36(76.6 \%)$ & $0.694^{\#}$ \\
\hline Blood sugar (mg/dL) & $135 \pm 62.0$ & $163 \pm 73.4$ & $127 \pm 56.4$ & $0.053^{*}$ \\
\hline
\end{tabular}

Continuous variables are presented as mean \pm SD.

${ }^{*}$ Student's t-test ${ }^{\#}$ Chi-square test

\section{ROC Curve}

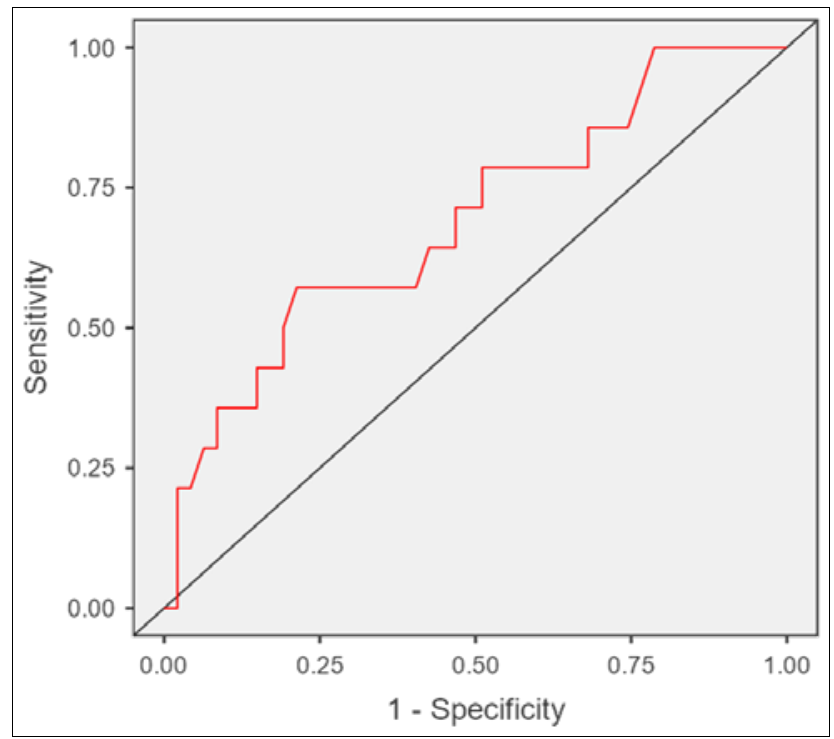

Fig 1: Receiver operating characteristic (ROC) curve of blood sugar level for detection of acute necrotizing pancreatitis.

\section{Discussion}

Acute pancreatitis is inflammation of pancreas with varied degrees of severity. This inflammatory process can activate enzymes in the pancreas further leading to auto digestion of this organ. After the initial insult due to various causes, gallstones being the most common one, pro-inflammatory cytokines such as interleukin (IL)-1, IL-2, IL-6, tumor necrosis factor- $\alpha$ (TNF$\alpha$ ) and anti-inflammatory mediators such as IL-10 and IL-1 receptor antagonist are released by the acinar cells. These propagate the response after the initial insult both locally and systemically. These further recruit TNF- $\alpha$, IL-1, IL-7, neutrophils, and macrophages, which in turn leads to release of more inflammatory mediators. Local inflammatory response in aggravated leading to damage to microcirculation. In severe cases this may cause pancreatic necrosis ${ }^{[4]}$.

Bank S et al. in 2002 concluded that a series of inflammatory reactions can be triggered due to inflammatory cytokines released in response to high level of glucose ${ }^{[5]}$. In the past there have been comparisons between glucose levels and, organ failure, ICU transfer rate and mortality rate. Ranson and Pasternack ascertained that blood glucose more than 200mg/dL is an indication of severe pancreatitis ${ }^{[6]}$. This was also added to the 11 grave prognostic signs which were known as Ranson signs ${ }^{[1]}$. Therefore, the relation between the severity and blood sugar levels has been talked about in the past. Moreover, it has also been said that high blood sugar can promote the release of inflammatory cytokines in acute pancreatitis ${ }^{[7]}$.

In our study, we found that blood sugar level has low sensitivity and positive predictive value for detection of pancreatic necrosis. However, it has acceptable specificity and good negative predictive value. Lankisch et al. had concluded in their paper published in 2001 that high blood glucose had sufficiently high sensitivity (83\%) and NPV (92\%) for detecting necrotizing pancreatitis. It had disappointing specificity and PPV (49 and $28 \%)^{[1]}$. This study agrees in aspect of NPV however has opposite results for sensitivity and specificity.

This signifies that blood sugar level of less than $140 \mathrm{mg} / \mathrm{dL}$ helps us rule out the presence of necrotizing gallstone induced acute pancreatitis. A negative test has more significance than a positive test. AUC of the ROC curve is also acceptable. Blood sugar analysis is a simple, quick and easily attainable investigation which can help us in the management of patients of acute gallstone induced pancreatitis. Excluding the possibility of necrotizing pancreatitis will let us take a less aggressive approach, for example, a CECT would not be required. So these patients could be managed at regional hospitals rather than tertiary care centers, which will prevent overburdening of valuable resources. 
There are some limitations to this study. First, sample size in this study was small and this study was only conducted in one institute. More studies are required with a larger sample size to ascertain the value of blood sugar levels in necrotizing acute pancreatitis. Second, this study was carried out in a tertiary care center in a hilly state where more severe cases are generally referred and managed. Therefore, this sample does not fully represent the cases in the general population.

However, this study had its strengths also. Not many studies have been conducted to look for relation between blood sugar and necrotizing pancreatitis. We have determined the value of blood sugar levels on arrival of the patient to the hospital, for ruling out presence of necrosis in the pancreas. These values were not altered by intravenous fluids. Also, all diabetic patients were excluded from the study which further added to the value of this investigation.

\section{Conclusion}

In conclusion, blood sugar levels bear significance in the management of patients of acute gallstone induced pancreatitis. They have been used in scoring systems for prediction of severity of acute pancreatitis. We infer that it also aids in determining absence of necrosis in acute pancreatitis. Therefore, may be used to rule out the possibility of acute necrotizing pancreatitis. This will help a lot especially where resources are limited and scarce. However, further studies are required to be absolutely certain in this regard.

\section{Acknowledgment}

We are thankful to all our patients who consented to participate in this study. We are also grateful to our institute 'Indira Gandhi Medical College, Shimla” for providing us a platform to carry out these studies.

Disclosure: The authors declare no conflict of interest.

\section{References}

1. Lankisch PG, Blum T, Bruns A, Dröge M, Brinkmann G, Struckmann $\mathrm{K}$ et al. Has blood glucose level measured on admission to hospital in a patient with acute pancreatitis any prognostic value? Pancreatology 2001;1(3):224-9. Doi: 10.1159/000055815.

2. Sarr MG. Revision of the Atlanta classification of acute pancreatitis. Pol Arch Med Wewn 2012-2013;123(3):11824. Doi: 10.20452/pamw.1627.

3. Yadav D, Lowenfels AB. The epidemiology of pancreatitis and pancreatic cancer. Gastroenterology 2013;144(6):125261. Doi: 10.1053/j.gastro.2013.01.068.

4. Elfar M, Gaber LW, Sabek O, Fischer CP, Gaber AO. The inflammatory cascade in acute pancreatitis: relevance to clinical disease. Surg Clin North Am 2007;87(6):1325-40. Doi: 10.1016/j.suc.2007.09.002.

5. Bank S, Singh P, Pooran N, Stark B. Evaluation of factors that have reduced mortality from acute pancreatitis over the past 20 years. J Clin Gastroenterol 2002;35(1):50-60. Doi: 10.1097/00004836-200207000-00012.

6. Bank S, Singh P, Pooran N, Stark B. Evaluation of factors that have reduced mortality from acute pancreatitis over the past 20 years. J Clin Gastroenterol 2002;35(1):50-60. Doi: 10.1097/00004836-200207000-00012.

7. Sun YF, Song Y, Liu CS, Geng JL. Correlation between the glucose level and the development of acute pancreatitis. Saudi J Biol Sci 2019;26(2):427-430. Doi: 10.1016/j.sjbs.2018.11.012. 